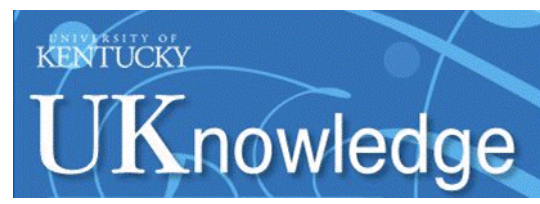

University of Kentucky

UKnowledge

6-2013

\title{
Volume Averaged Modeling of the Oxidation of Porous Carbon Fiber Material
}

\author{
Alexandre Martin \\ University of Kentucky, alexandre.martin@uky.edu
}

Follow this and additional works at: https://uknowledge.uky.edu/me_facpub

Part of the Aerodynamics and Fluid Mechanics Commons, Computer Sciences Commons, and the Space Vehicles Commons

Right click to open a feedback form in a new tab to let us know how this document benefits you.

\section{Repository Citation}

Martin, Alexandre, "Volume Averaged Modeling of the Oxidation of Porous Carbon Fiber Material" (2013). Mechanical Engineering Faculty Publications. 13.

https://uknowledge.uky.edu/me_facpub/13

This Conference Proceeding is brought to you for free and open access by the Mechanical Engineering at UKnowledge. It has been accepted for inclusion in Mechanical Engineering Faculty Publications by an authorized administrator of UKnowledge. For more information, please contact UKnowledge@lsv.uky.edu. 


\section{Volume Averaged Modeling of the Oxidation of Porous Carbon Fiber Material}

Digital Object Identifier (DOI)

http://dx.doi.org/10.2514/6.2013-2636

\section{Notes/Citation Information}

Published in the Proceedings of the 44th AIAA Thermophysics Conference, Paper 2013-2636, p. 1-11.

Copyright @ 2013 by Alexandre Martin.

The copyright holder has granted the permission for posting the article here. 


\title{
Volume averaged modeling of the oxidation of porous carbon fiber material
}

\author{
Alexandre Martin* \\ University of Kentucky, Lexington, KY, 40506, USA
}

\begin{abstract}
Charring ablators remain the premium choice for space exploration missions that involve atmospheric re-entry. This type of ablative material is composed of a carbon matrix, usually made of fibers, which is then impregnated with a resin. During re-entry, the high heat flux produced by convective heating causes the material to chemically react. First, the resin pyrolyzes, and is vaporized into a gas that travels through the material, and is eventually ejected at the surface. Then, as the temperature rises, the surface of the porous matrix recess through ablative processes. For re-entry conditions typical of space exploration missions, this is mainly diffusion limited oxidation. However, recent studies have shown that oxygen from the atmosphere actually penetrates a thin layer of the porous material, oxidizing the carbon fibers from within.

This research activity presents a volume-averaged fiber-scale oxidation model, based on the one previously developed by Lachaud et al. The present model, however, solves the momentum equation as well as the energy equation. Results based on a an experimental test case are presented. The importance of solving both equations is clearly demonstrated, and a new value for carbon fiber oxidation reactivity is suggested.
\end{abstract}

\section{Introduction}

For extra-orbital missions, charring ablative materials have always been, and still remain, the primary choice for the design of atmospheric re-entry heat shields. These materials are usually made of a carbon matrix, typically composed of micro fibers, impregnated with a pyrolyzing resin, usually phenol.

With this type of material, the convective heating transmitted to the surface is dissipated by thermal and chemical decomposition. The gas emitted from the inner decomposition of the matrix is expelled into the free stream, thickening the boundary layer and pushing the bow shock away from the surface. This creates an additional protective gas layer at the surface but, more importantly, the chemical composition at the surface of the vehicle is changed considerably, which also attenuates the transmitted heat by modifying the thermal conductivity of the boundary layer, and allowing endothermic chemical reactions in that region.

The composition of the boundary layer also greatly influences surface reactions. For charring ablators, it has been speculated that surface reaction does not occur on the surface of the material, but in a thin layer near the surface. ${ }^{1}$ The ablator in this region entirely pyrolyzes, leaving the carbon completely exposed to the surrounding gas. Because of the high porosity of the material, reacting gas from the outer flow, mostly oxygen, diffuses inside the layer, and reacts at the surface of the fibers, eroding them until they completely vanish.

In this context, it is evident that there is a blatant need to update and modernize past experiments, such as the one presented in Ref. 2, and propose new, validated pyrolysing chemistry and surface reaction models. In the last year, the first steps of such a study has been underway, ${ }^{3}$ and it is hopeful that this effort will lead to a more accurate description of the behavior of the chemically reacting pyrolysis gas, as well as fiber erosion. In the meantime, the current paper aims at preparing the numerical framework necessary to evaluate and complement the outcome of these experiments. In order to do this, a validated material response code was recently modified to account for homogeneous chemical non-equilibrium for the pyrolysis gas, and heterogeneous reaction of the gas with the surface of the carbon fibers. ${ }^{4}$ Using these new features,

*Assistant Professor, Department of Mechanical Engineering, Associate Faculty - Center for Computational Science, Senior Member AIAA. Email: alexandre.martin@uky.edu 
this paper proposes to take a closer look at the effects that a varying temperature has on the reactivity of the carbon fibers.

\section{Material response code}

\section{Chemical equilibrium material response}

The material response code used in the present study has been developed and validated over the last few years, ${ }^{5}$ and was recently adapted to account for nonequilibrium chemistry. ${ }^{4}$ The code models gas flow through porous media by solving the following four conservation equations in one dimension:

Mixture Energy Equation

$$
\frac{d}{d t} \int_{c v} \rho E d V-\int_{c s} \rho h v_{c s} d S+\int_{c s} \phi \rho_{g} h_{g} v_{g} d S+\int_{c s} \dot{q}^{\prime \prime} d A+\int_{c s} \phi \sum_{k} J_{k} h_{g_{k}} d A=0
$$

Solid Phase Equation

$$
\frac{d}{d t} \int_{c v} \rho_{s} d V-\int_{c s} \rho_{s} v_{c s} d S-\int_{c v} \dot{m}_{s}^{\prime \prime \prime} d V=0
$$

Gas Phase Continuity Equation

$$
\frac{d}{d t} \int_{c v} \phi \rho_{g k} d V-\int_{c s} \phi \rho_{g k} v_{c s} d S+\int_{c s} \phi \rho_{g k} v_{g} d S-\int_{c s} \phi J_{k} d S-\int_{c v}\left(\dot{m}_{g k}^{\prime \prime \prime}+\omega_{k}\right) d V=0
$$

Momentum Equation: Darcy's Law

$$
\frac{\partial P}{\partial z}=-\frac{\mu}{K} \phi v_{g}
$$

The first terms in Eqs. (1) to 3 account, respectively, for the energy, solid mass, and gas mass content, and the second term for the grid convection. The third terms in Eqs. 1 and 3 account for the gas flux, and the last terms in Eqs. 2 to 3, the source term. As for the fourth term of Eq. 1, it accounts for the heat conduction, and it is modeled according to Fourier's Law:

$$
\dot{q}^{\prime \prime}=-\kappa \frac{\partial T}{\partial z}
$$

The kinetic energy $\tilde{E}_{k i n}$ of the pyrolysis gas, which is usually negligible in most material response code applications, becomes quite important in this simulation. Usually, the gas phase contribution to the energy equation is a few orders of magnitude less than the terms related to the solid material, mostly because of the great difference in density and heat capacity. However, in the present application,the oxidation gas is modeled before and after it enters the porous sample, in which case, the kinetic energy contribution is of the same order of magnitude as the other terms. The kinetic energy is therefore calculated using:

$$
\tilde{E}_{k i n}=\frac{d}{d t} \int_{c v} \rho \frac{v_{g}^{2}}{2} d V+\int_{c s} \phi \rho_{g} \frac{v_{g}^{2}}{2} v_{g} d A-\int_{c s} \rho \frac{v_{g}^{2}}{2} v_{c s} d A
$$

In theory, Darcy's law is only valid for porous flow. However, it is possible to adapt it for non-porous media by adjusting the value of permeability. Since the average velocity of a steady, laminar and fully developed flow in a pipe of radius $R$ is given by

$$
\bar{v}=-\frac{\partial P}{\partial z} \frac{R^{2}}{8 \mu}
$$

Using Eq. 4, it can be seen that for a flow inside a tube, for which $\phi=1.0$, since there is no porous material, Darcy's formulation can be used by simply setting the permeability to $K=R^{2} / 8$. 


\section{Nonequilibrium chemistry}

In the gas conservation equation (Eq. 3), $k$ represents one of the species of the finite rate chemistry model; there is therefore such an equation for all the species considered, and $\sum_{k} \rho_{g k}=\rho_{g}$. The term $\dot{m}_{g k}^{\prime \prime \prime}$ relates to the mass fraction of the solid that is transferred to species $k$, and $\omega_{k}$ relates to the amount of species $k$ that is created using the finite-rate chemistry model. The latter source term is evaluated by:

$$
\omega_{k}=M_{k} \sum_{j=1}^{m}\left(\beta_{k j}-\alpha_{k j}\right)\left[k_{f j} \prod_{i=1}^{q}\left(\frac{\rho_{i}}{M_{i}}\right)^{\alpha_{i j}}-k_{r j} \prod_{i=1}^{q}\left(\frac{\rho_{i}}{M_{i}}\right)^{\beta_{i j}}\right]
$$

where $\alpha_{i j}$ and $\beta_{i j}$ are, respectively, the stoichiometric coefficients for product and reactants $i$ of reaction $j$ and $M_{j}$ the molar mass of species $j$. The forward $k_{f}$ and backward $k_{r}$ rates are calculating using an Arrhenius equation:

$$
k_{f, b}=k_{0} T^{-s} \exp (-E / R T)
$$

in which the coefficients $k_{0}, s$ and $E$ are the typical Arrhenius coefficients. The species mass diffusion, $J_{k}$, is modeled using Fick's Law:

$$
J_{k}=-\rho \frac{D_{k}}{\eta} \frac{\partial Y_{k}}{\partial z}
$$

where $Y_{l}$ is the mass fraction of species $k$, and $\eta$ the tortuosity. This equation is implemented in a way that enforces that the sum of all the diffusion fluxes are zero, if the models used to calculate the species diffusion $D_{k}$ require it. ${ }^{6}$ A mass diffusion term is also present in the energy equation.

\section{Thermodynamical and Transport properties}

The chemical properties of each individual species are evaluated using the thermodynamic curve fits obtained from Ref. 7. This provides a value for the heat capacity $C_{P k}$ of each species, as well as enthalpy $h_{k}$, heat of formation $h_{k}^{0}$ and entropy $S_{k}$. These values are also used to calculate the equilibrium values if reversible reactions are used in the finite-rate chemistry model. The single species viscosity $\mu_{k}$ and conductivity $\kappa_{k}$ are obtained using curve fits compiled in Ref. 8. Because of the lack of data available for multi-component viscosity, the property of the gas mixture is evaluated using the simple approximation to the first term of the Chapman-Enskog expansion, Wilke's mixing rule: ${ }^{9}$

$$
\mu=\sum_{k} \frac{X_{k} \mu_{k}}{\phi_{k}} \text { and } \kappa=\sum_{k} \frac{X_{k} \kappa_{k}}{\phi_{k}}
$$

where $X_{k}$ is the molar fraction and $\phi_{k}$ is given by:

$$
\phi_{k}=\sum_{r} X_{r} \frac{\left[1+\sqrt{\left.\frac{\mu_{k}}{\mu_{r}}\left(\frac{M_{r}}{M_{k}}\right)^{1 / 4}\right]^{2}}\right.}{\sqrt{8\left(1+\frac{M_{k}}{M_{r}}\right)}}
$$

Because no binary collision data is used in this approach, the diffusion coefficient $D_{k}$ is obtained using the constant Lewis Number approximation:

$$
D_{k}=D=\frac{\text { Le } k}{\rho C_{P}}
$$

\section{Volume-averaged fiber-scale oxidation modeling}

It has been shown that the so-called surface ablation, is more likely to be a volumetric phenomenon. ${ }^{1}$ Lachaud has theorized, and later demonstrated, that the oxygen from the surrounding flow actually penetrates the porous material over small distance, and oxidize the material from within. The recession rate, therefore, is not based on the macroscopic surface that is exposed to the flow field, but on the surface of the carbon fibers, and thus the porosity of the material. In this study, the chemical oxidation is therefore only modeled by using a set of heterogeneous reaction:

$$
\mathrm{C}_{(\mathrm{s})}+\mathrm{O}_{2} \longrightarrow \mathrm{CO}_{2}
$$




$$
\begin{aligned}
& \mathrm{C}_{(\mathrm{s})}+1 / 2 \mathrm{O}_{2} \longrightarrow \mathrm{CO} \\
& \mathrm{C}_{(\mathrm{s})}+\mathrm{CO}_{2} \longleftrightarrow 2 \mathrm{CO}
\end{aligned}
$$

In the range of interest for an ablating charring ablator, all three of these reactions are important. The third reaction, the so called 'Boudouard reaction", dictates the ratio of $\mathrm{CO} / \mathrm{CO}_{2}$ created during the oxidation process (gasification). Below $973 \mathrm{~K}$, the Boudouard equilibrium temperature, ${ }^{10}$ mostly $\mathrm{CO}_{2}$ is produced. Conversely, $\mathrm{CO}$ is the dominating product when the temperature is higher than $973 \mathrm{~K}$. If the temperature oscillates around that equilibrium point, an interesting phenomenon may occur. As the gas cools down, the transformation of the $\mathrm{CO}$ into $\mathrm{CO}_{2}$ leads to soot formation. This process has been observed in post flight analysis of porous ablators. The gases formed by these heterogeneous reactions are also expected to interact in the gas phase. Depending on the temperature, various exchange reactions between $\mathrm{N}_{2}, \mathrm{O}_{2}, \mathrm{CO}_{2}$ and $\mathrm{CO}$ are likely to occur.

For this study, the chosen test case uses a temperature of $898 \mathrm{~K}$ : therefore, only the first oxidation reaction is expected to matter, and no gas phase kinetics are expected to take place. The reaction rate is expressed as the parameter $k_{f}$, and the diffusion flux of incoming oxygen is equivalent to the flux of outgoing carbon dioxide:

$$
-J_{\mathrm{CO}_{2}}=J_{\mathrm{O}_{2}}=k_{f} \chi_{\mathrm{O}_{2}}
$$

where $\chi_{\mathrm{O}_{2}}$ is the molar concentration of oxygen. Thus, the surface recession of an individual fiber of radius $r$ is expressed in terms of the molar diffusion of carbon dioxide $J_{\mathrm{CO}_{2}}$ at the surface:

$$
\dot{\mathbf{r}}=\Omega J_{\mathrm{CO}_{2}} \mathbf{n}
$$

where $\Omega=M_{c} / \rho_{c}$ is the solid molar mass of the carbon, and $\mathbf{n}$ the normal vector to the surface of the fiber, pointing outward. Assuming that the fibers are perfectly cylindrical, and that they recess uniformly, this equation can be expressed in $1 \mathrm{D}$ as:

$$
\frac{d r}{d t}=-\Omega k_{f} \chi_{\mathrm{O}_{2}}
$$

The volumetric approach used here assumes that the fibers enclosed in a control volume $V_{T}$ all have the same initial radius, and are distributed homogeneously. The control volume can therefore be split into the volume occupied by the fibers $V_{f}$, and the volume "occupied" by the pores $V_{p}$. These can be expressed in terms of volume fraction:

$$
\frac{V_{f}}{V_{T}}+\frac{V_{p}}{V_{T}}=\epsilon+\phi=1
$$

where $\epsilon$ is the fiber volume fraction, and $\phi$ the porosity. The volume occupied by $N$ fibers of diameters of diameter $r$ and length $l_{f}$ are therefore given by $V_{f}=N \pi r^{2} l_{f}$. As the fibers oxidize, the fiber volume fraction changes, and can be expressed as a function of the initial volume fraction:

$$
\epsilon=\epsilon_{0} \frac{r^{2}}{r_{0}^{2}}
$$

. In order to be included in the material response code, these relations have to be expressed in terms of the bulk density of the carbon matrix. This density can be expressed in terms of the mass of all fibers enclosed in the control volume:

$$
\rho_{s}=\frac{m_{f}}{V_{T}}=\frac{N \pi r^{2} l_{f}}{V_{T}} \rho_{\mathrm{C}}=\epsilon \rho_{\mathrm{C}}
$$

where $\rho_{\mathrm{C}}$ is the density of solid carbon (not to be confused with the bulk density). The oxidation of fibers, expressed in terms of bulk density change, is therefore:

$$
\frac{\partial \rho_{s}}{\partial t}=-\frac{\epsilon_{0}}{r_{0}^{2}} 2 r \rho_{\mathrm{C}} \frac{\partial r}{\partial t}
$$

By using the parameter $S_{f}$, which represents the volumetric surface of occupancy by the fibers, and is defined as

$$
S_{f}=\frac{N \pi 2 R l_{f}}{V_{T}}=2 \frac{\epsilon_{0}}{r_{0}^{2}} r
$$


as well as Eq. 6, this equation becomes:

$$
\frac{\partial \rho_{s}}{\partial t}=-k_{f} S_{f} M_{\mathrm{C}} \chi_{\mathrm{O}_{2}}
$$

or, if re-arranged in terms of $\rho_{s}$ :

$$
\frac{\partial \rho_{s}}{\partial t}=-2 k_{f} \frac{M_{\mathrm{C}}}{M_{\mathrm{O}_{2}}} \frac{\rho_{\mathrm{O}_{2}}}{r_{0}} \sqrt{\frac{\epsilon_{0}}{\rho_{\mathrm{C}}}} \sqrt{\rho_{s}}
$$

This expression can be solved analytically to give the evolution of the bulk density over time step $\Delta t$ :

$$
\rho_{s}^{(t)}=\left({\sqrt{\rho_{s}}}^{(t-1)}-k_{f} \frac{M_{\mathrm{C}}}{M_{\mathrm{O}_{2}}} \frac{1}{r_{0}} \sqrt{\frac{\epsilon_{0}}{\rho_{\mathrm{C}}}} \int_{(t-1)}^{(t)} \rho_{\mathrm{O}_{2}} d t\right)^{2}
$$

The account of oxidation on the gas phase cannot be solved analytically, and must be integrated as a source term in Eq. 3:

$$
\dot{m}_{\mathrm{O}_{2}}^{\prime \prime \prime}=-\rho_{\mathrm{O}_{2}} \frac{M_{\mathrm{O}_{2}}}{M_{\mathrm{O}_{2}}} S_{f} k_{f}, \quad \dot{m}_{\mathrm{CO}_{2}}^{\prime \prime \prime}=\rho_{\mathrm{O}_{2}} \frac{M_{\mathrm{CO}_{2}}}{M_{\mathrm{O}_{2}}} S_{f} k_{f}
$$

It is to be noted that Eq. 8 can be re-written in the same form, to give:

$$
\dot{m}_{\mathrm{C}}^{\prime \prime \prime}=-\rho_{\mathrm{O}_{2}} \frac{M_{\mathrm{C}}}{M_{\mathrm{O}_{2}}} S_{f} k_{f}
$$

\section{Numerical approach}

The code solves Eqs. 3 and 1 implicitly on an arbitrary contracting grid employing Landau coordinates. Equation 2 is straightforward, and does not need to be solved numerically. As for Darcy's law, Eq. 4, it is explicitly solved for $v_{g}$ and directly integrated in the gas-phase continuity equation.

Newton's method for nonlinear systems is used to solve each of the equations, and an iterative process is performed over the whole set until convergence is attained. This method, called block Gauss-Seidel, converges linearly, and is quite efficient when applied to a reduced set of equations. In the chemical equilibrium version of the code, only two equations needed to be solved numerically, and that method is appropriate. In the chemical non-equilibrium version, the number of equations is dependent on the number of species, and therefore increases the iterative process immensely. Instead of using block Gauss-Seidel on each of the $1+N_{S}$ equations, where $N_{S}$ is the total number of species, the method is only applied to the energy and the total gaseous mass conservation equation. However, in this second equation, the mass conservation of each species is solved at once, using the Newton method. This method requires the inversion of a block tri-diagonal system of equations, instead of a simple tri-diagonal.

To account for fiber oxidation, Eq. 9 is solved directly over time step $\Delta t$ to calculate the solid decomposition and the surface function $S_{f}$. The latter quantity is then used as source terms in the gas phase equation (Eq. 3) by way of Eq. 10.

\section{Problem description}

The material response code has been adapted to model a very specific set of experiments performed at the NASA Ames sidearm reactor facility, ${ }^{3}$ which, in this very specific case, is only used as a high temperature flow tube reactor. The test-case presented here reproduces the experimental conditions for one example in a series of planned experiments which will take place in the near future. The flow tube reactor, illustrated in Fig. 1, consist of a $2.2 \mathrm{~cm}$ diameter tube in which a FiberForm sample is introduced. The sample, $2.03 \mathrm{~cm}$ in length, completely fills the tube radially, which forces the gas through it. Before reaching the sample, the gas travels within a long pipe which ensure that it reaches a fully developed state, and that the temperature is not fluctuating. The specific case modeled here has the gas traveling at a mass flow rate of $2.149 \times 10^{-6} \mathrm{~kg} / \mathrm{s}$, with a pressure at the inlet of $12.8 \mathrm{kPa}$. The pressure at the back of the sample is not fixed, and is constantly adjusted to ensure that the inlet conditions are preserved. The pressure at the outlet is measured during the experiments, and therefore provides an addition comparison point with the simulation. The initial porosity

of FiberForm has been measured at 0.9 , and the bulk density to $184 \mathrm{~kg} / \mathrm{m}^{3}$. The tortuosity is set to 1.15 for the virgin state of the sample, and decreases linearly to 1.00 when the material reaches a porosity of 1.0 . 


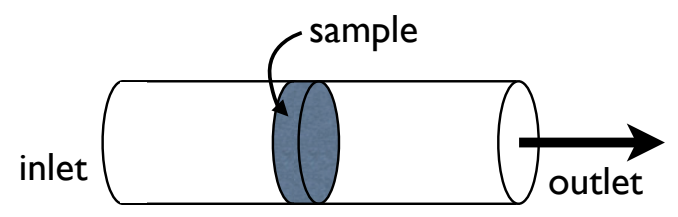

Figure 1. Geometry of the test section of the NASA Ames flow tube reactor

The permeability is evaluated at $1.367 \times 10^{-10}$ for the virgin FiberForm, and decreases exponentially to the free stream value as the porosity linearly increases. As mentioned earlier, because the code uses Darcy's law to model the momentum transport, this would translate into permeability of $1.5125 \times 10^{-5} \mathrm{~m}^{2}$. However, such a high value of the permeability considerably raises the numerical stiffness of the code, and prevents the solution to be obtained in a timely manner. However, since both the mass flow rate and the pressure are imposed at the inlet, and because the gas velocity is relatively slow (Reynolds number of 2-3), it can easily be shown that using a smaller permeability, even 2 or 3 orders of magnitude smaller, only affects the pressure in the flow tube, and not by very much. Fig 2 shows the mass flow rate through the sample, using various permeability values, for a non-reacting sample. As can be seen, the difference in pressure is not significant until the permeability reaches a value of the order of $10^{-7} \mathrm{~m}^{2}$.

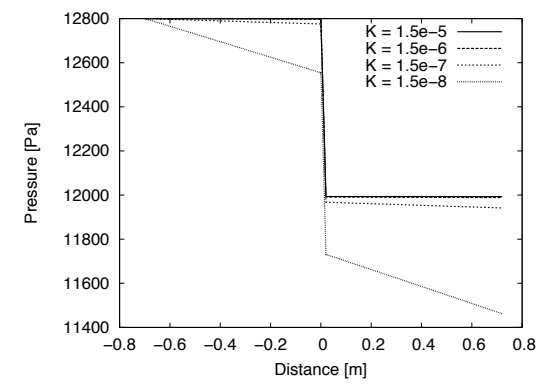

(a) Pressure

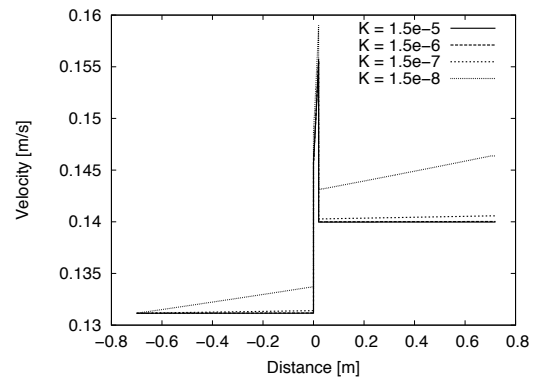

(b) Velocity

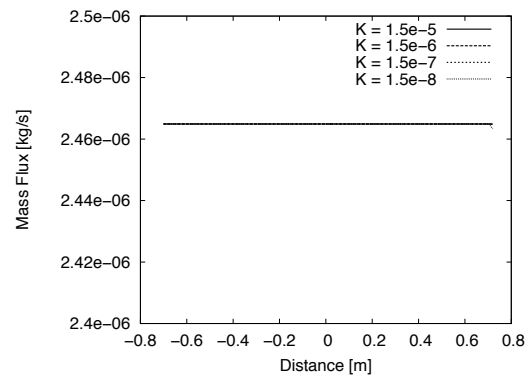

(c) Mass flux

Figure 2. Pressure, velocity and mass flux distribution of the steady state solution of the flow tube reactor, using various permeability values to model the free stream. The $2.03 \mathrm{~cm}$ sample is non-reacting, and is located at $x=0.0$, where the pressure drops and velocity rise occurs.

Another key aspect of the simulation is that a section of the tube must be modeled in order for a reasonable diffusion driven boundary layer to develop in front of the sample. This is necessary as the flow travels at such a small velocity (around $0.114 \mathrm{~m} / \mathrm{s}$ ) that the diffusion velocity is not negligible. This means that, as the sample oxidizes, a significant amount of $\mathrm{CO}_{2}$ will diffuse in the opposite direction of the flow, therefore reduce the amount of available $\mathrm{O}_{2}$. It is clear that the accuracy of the diffusion coefficients plays a significant role in the surface recession. It was previously shown ${ }^{4}$ that the absence of such a buffer zone causes significant changes in the results, going from surface recession overestimation to flow reversal. For different reasons, it is also imperative to model a section of the tube after the sample. This allows the energy released by the oxidation chemistry to be properly convected away by the flow, and not remain in the test-article. The importance of this modeling aspect was also demonstrated in Ref. 4. For these reasons, a free stream tube length of 0.7 meter is modeled in front and at the back of the sample.

Finally, a re-radiating source term is apply at both end of the sample, which mitigates increases of energy within the sample.

\section{Results}

Results of the full simulation are presented in Fig. 3, using a reactivity of $k_{f}=0.01 \mathrm{~m} / \mathrm{s}$, which is the one reported in Ref. 3. The results present a quantitative match with the experimental recession rate of 7 $\mathrm{mm}$. 


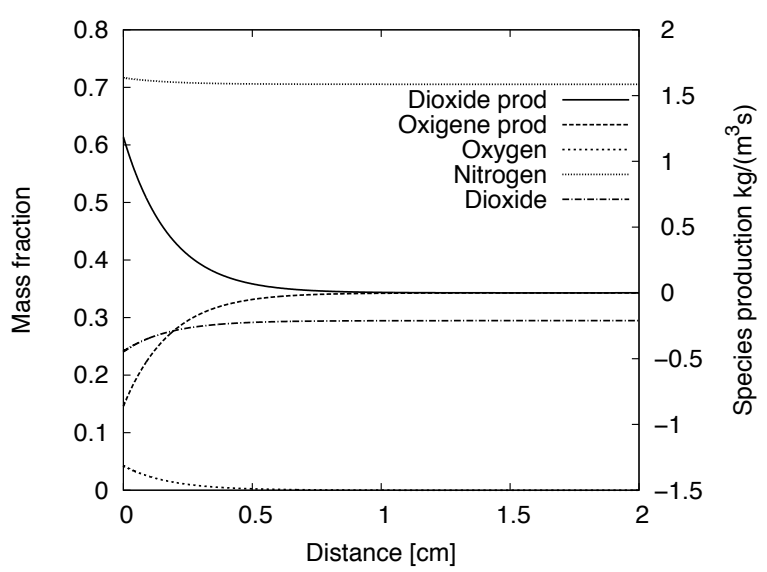

(a) 1 seconds

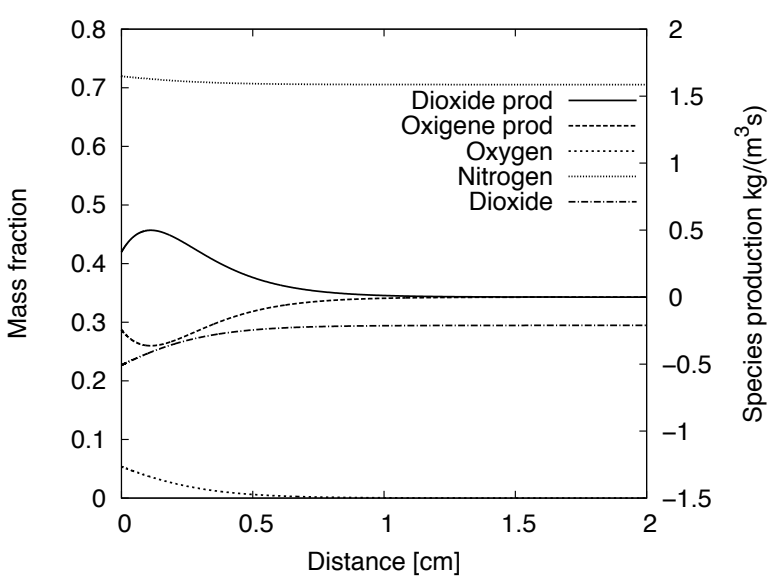

(c) 15 minutes

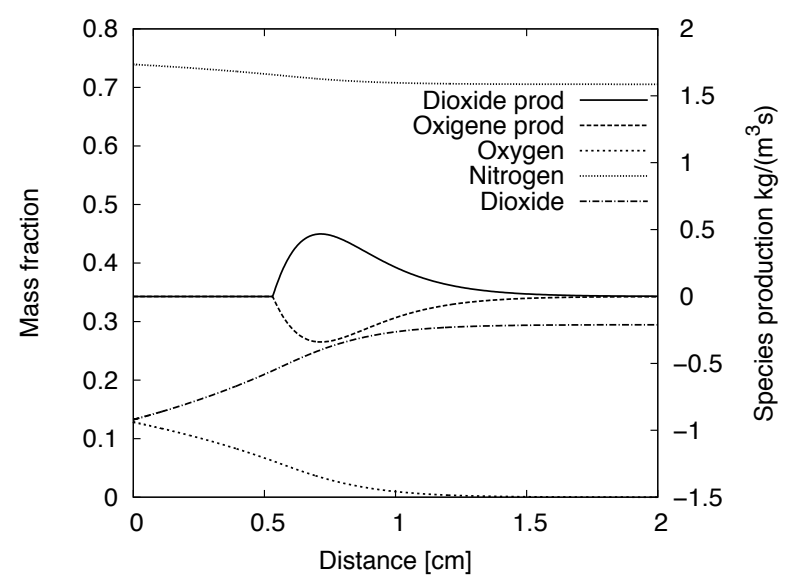

(e) 50 minutes

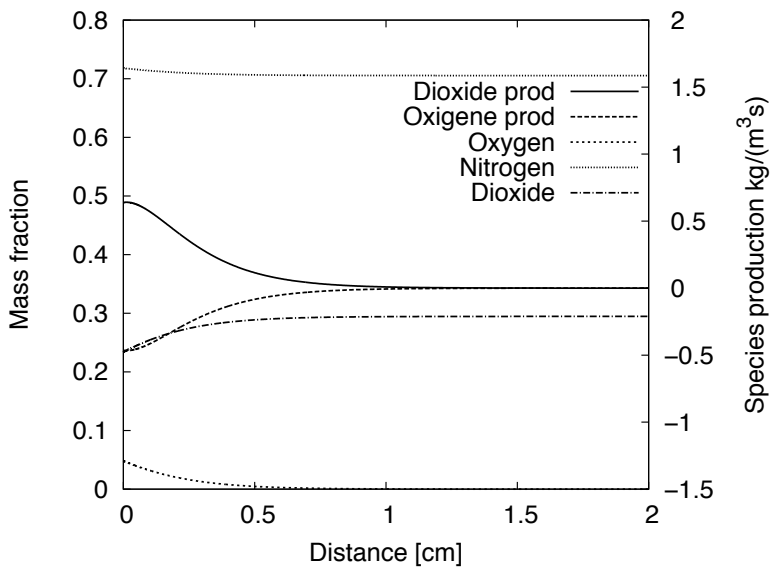

(b) 10 minutes

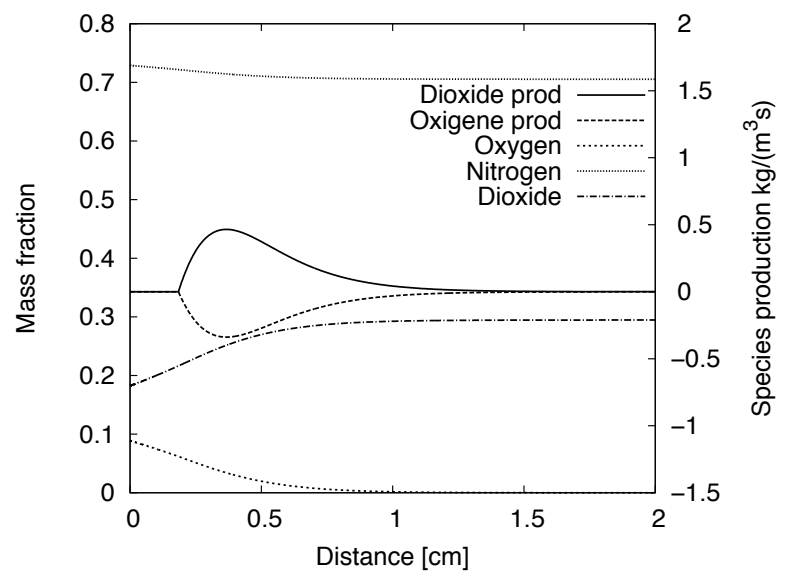

(d) 30 minutes

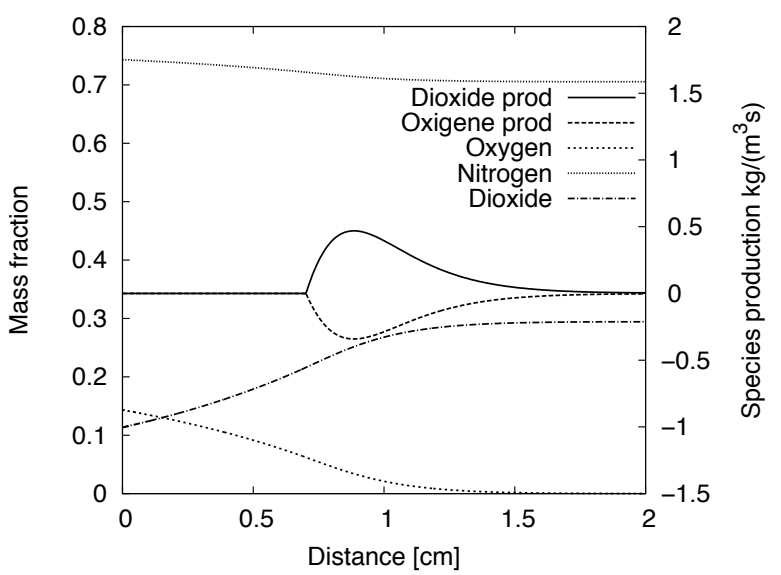

(f) 60 minutes

Figure 3. Time dependent gas mass fraction and species production rate inside the FiberForm sample for a mass flow rate of $2.149 \mathrm{mg} / \mathrm{s}$ and a fixed fiber reactivity of $k_{f}=0.01 \mathrm{~m} / \mathrm{s}$. 


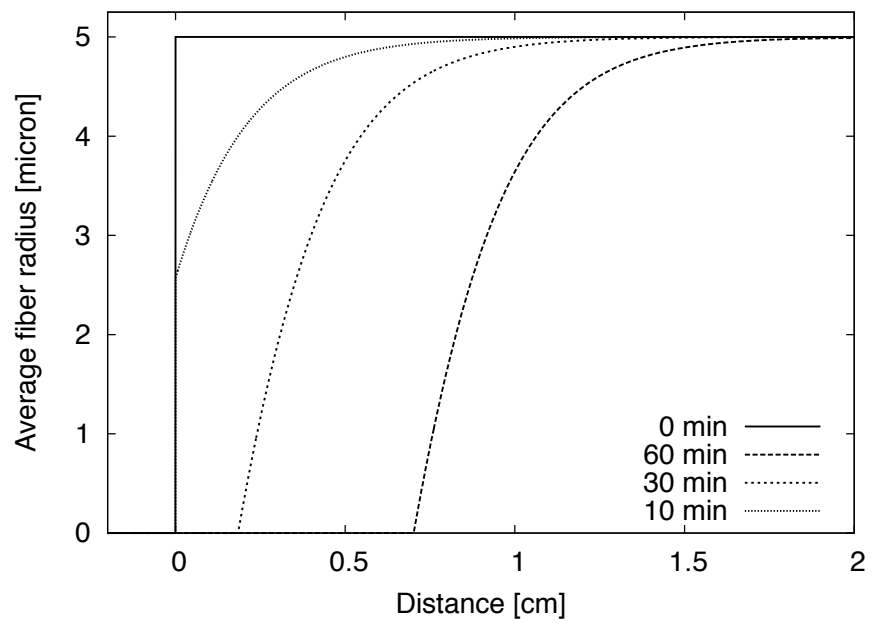

Figure 4. Time dependent Fiber diameter inside the FiberForm sample for a mass flow rate of 2.149 mg/s and a fixed fiber reactivity of $k_{f}=0.01 \mathrm{~m} / \mathrm{s}$.

First, it is interesting to look at the oxygen penetration. Since the presented model solves the momentum equation, the gas velocity is calculated, and not inferred from the incoming mass flow rate. As can be seen on Fig. 4 oxygen is therefore pushed in much deeper then the $2 \mathrm{~mm}$ reported in Ref. 3.

Since the code also solves the energy equation, results for the temperature of the gas are also presented. Because oxidation is an exothermic process, a rise in temperature is expected. As shown on Fig. 5, this rise is significant, and means that the temperature of the sample, at which the reaction occurs, is not the same as the one at the inlet. This rise in temperature could explain why the reactivity reported in Ref. 3 is higher than expected. It is also important to point out is that these results are significantly different than the ones presented in Ref. 4, now that the outlet boundary condition is treated correctly.

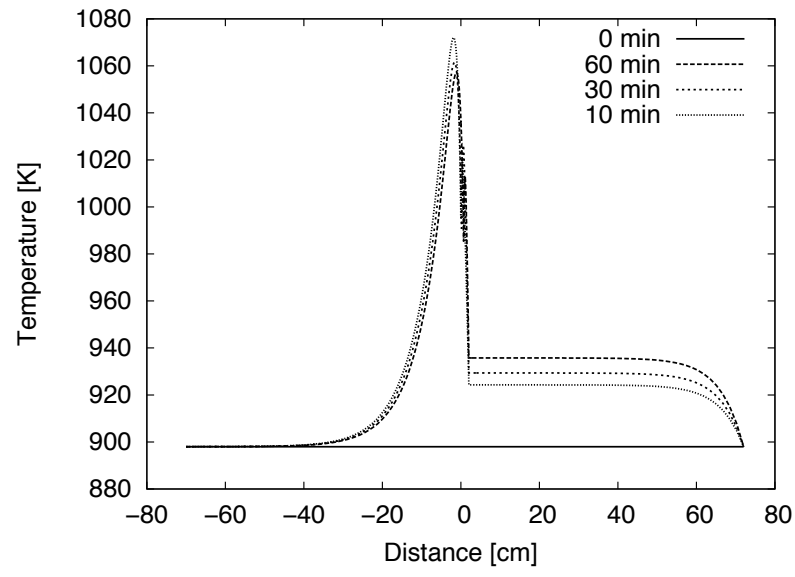

(a) Inside the flow tube reactor

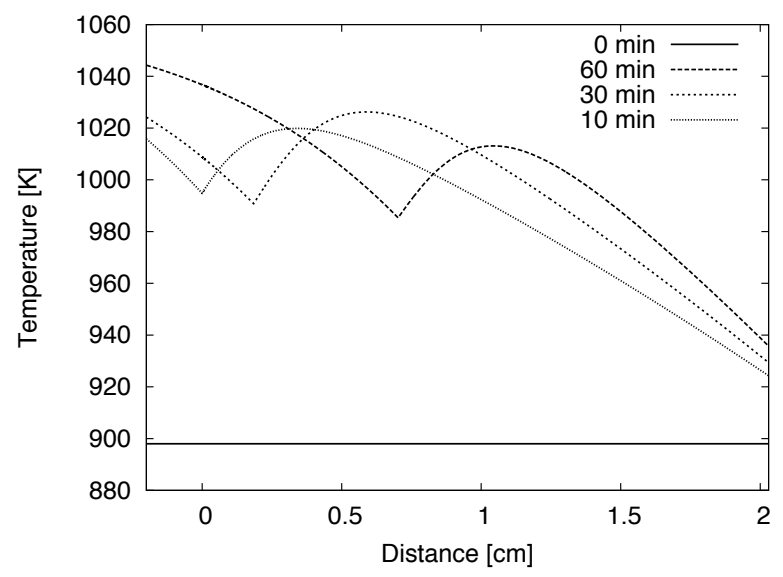

(b) Inside the FiberForm sample

Figure 5. Temperature as a function of time, with a fixed fiber reactivity of $k_{f}=0.01 \mathrm{~m} / \mathrm{s}$

In order to account for that rise in temperature, the reactivity of the fibers is modified according to an Arrhenius relation:

$$
k_{f}=A_{f} e^{-E_{a} /(R T)}
$$

where $E_{a} \approx 120 \mathrm{~kJ} / \mathrm{mol}$ and $A_{f}=10^{5} \mathrm{~m} / \mathrm{s}$. At a $T=898 \mathrm{~K}, k_{f}$ is approximately $0.01 \mathrm{~m} / \mathrm{s}$, which is the same value used in the previous results. As can be seen in Fig. 6, the end results are quite different. Since the fibers become more reactive as the temperature rises, the recession rate is higher, the $\mathrm{CO}_{2}$ production higher, and, consequently, the oxygen penetration lower. These results are more in line with the post-experiment 
microscopic observations; however, the recession rate, presented in Fig 7, no longer matched the experimental value of $0.7 \mathrm{~cm}$. It is therefore safe to conclude that the value of $A_{f}$ needs to be greater than the $10^{5} \mathrm{~m} / \mathrm{s}$ used here. As for the temperature inside the sample, presented on Fig 8, it shows no significant difference from the fixed reactivity results, although the values are slightly higher, and more uniform over time. However, this graphs clearly shows that the gas is at a higher temperature than the Boudouard equilibrium point (973 $\mathrm{K}$ ) when inside the sample, and goes below it as it exits. This means that soot is probably formed in the back part and behind the sample. The presence of carbon particles has been observed in the preliminary experiments, although it difficult today with any certitudes that they were soot particles.

From these results, it is clear that both the momentum equation and the energy equation must be solved to capture the physics of the problem. It is also likely that axisymmetric effects are importance, since the flow travels through the pipe at various axial velocities, and that the gas diffuses in all direction equally, when in the boundary layer. Moreover, since the flow tube is kept at a constant temperature, the temperature distribution inside the ablator will vary with the radius, as will the recession. This variable recession rate, however, was not reported in Ref. 3 .

\section{Conclusion}

A material response code modified according to a volume averaged approached was used to evaluate the oxidation reactivity of a porous, fibrous carbon test sample. This approach showed significant improvement in terms of surface recession capabilities. Building on a previously developed model, ${ }^{3}$ the new model also solves the energy and momentum equations. The results show good agreement with experiments and prior results. More importantly, they clearly demonstrate the importance of accounting for momentum and energy when modeling such a problem. This indicates that the formulation of a temperature dependent reactivity empirical relation for fibrous carbon oxidation will require more complex calculations.

\section{Acknowledgments}

Financial support for this work was provided by NASA SBIR Phase-2 Award NNX10CC53P, and NASA Kentucky EPSCoR Award NNX10AV39A. The author would like to thank Dr. Francesco Panerai and Dr. Sean Bailey at the University of Kentucky, as well as Dr. Jean Lachaud, at NASA Ames Research Center, for several useful discussions.

\section{References}

\footnotetext{
${ }^{1}$ Lachaud, J. and Mansour, N. N., "Microscopic scale simulation of the ablation of fibrous materials," 48th AIAA Aerospace Sciences Meeting and Exhibit, No. AIAA 2010-984, Orlando, FL, 4-7 January 2010. doi:10.2514/6.2010-984

${ }^{2}$ April, G. C., Energy transfer in the char zone of a charring ablator, Ph.D. thesis, Louisiana State University, Baton Rouge, LA, May 1st 1969.

${ }^{3}$ Lachaud, J. R., Mansour, N. N., Ceballos, A., Pejaković, D., Zhang, L., and Marschall, J., "Validation of a volumeaveraged fiber-scale model for the oxidation of a carbon-fiber preform," 42nd AIAA Thermophysics Conference, No. AIAA 2011-3640, Honolulu, HI, 27-30 June 2011. doi:10.2514/6.2011-3640

${ }^{4}$ Martin, A., "Modeling of chemical non equilibrium effects in a charring ablator," 51st AIAA Aerospace Sciences Meeting, No. AIAA 2013-0301, Grapevine, TX, January 7-10 2013. doi:10.2514/6.2013-301

${ }^{5}$ Martin, A. and Boyd, I. D., "Non-Darcian behavior of pyrolysis gas in a thermal protection system," Journal of Thermophysics and Heat Transfer, Vol. 24, No. 1, Jan.-Mar. 2010. doi:10.2514/1.44103

${ }^{6}$ Sutton, K. and Gnoffo, P. A., "Multi-component diffusion with application to computational aerothermodynamics," $7 t h$ AIA A/ASME Joint Thermophysics and Heat Transfer Conference, No. AIAA 1998-2575, Albuquerque, NM, June 15-18 1998. doi:10.2514/6.1998-2575

${ }^{7}$ McBride, B. J., Gordon, S., and Reno, M. A., "Coefficients for calculating thermodynamic and transport properties of individual species," Technical Memorandum TM-451, NASA, 1993.

${ }^{8}$ Scoggins, J. B., The Development of a Thermochemical Nonequilibrium Ablation and Pyrolysis Model for CarbonPhenolic Thermal Protection Systems, Master's thesis, North Carolina State University, 2011.

${ }^{9}$ Wilke, C., "A viscosity equation for gas mixtures," J. Chem. Phys., Vol. 18, No. 4, 1950, pp. 517-519. doi:10.1063/1.1747673

${ }^{10}$ Ellingham, H. J. T., "Reducibility of Oxides and Sulfides in Metallurgical Processes," Journal of the Society of Chemical Industry, Vol. 63, No. 5, 1944, pp. 125.

doi:10.1002/jctb.5000630501
} 


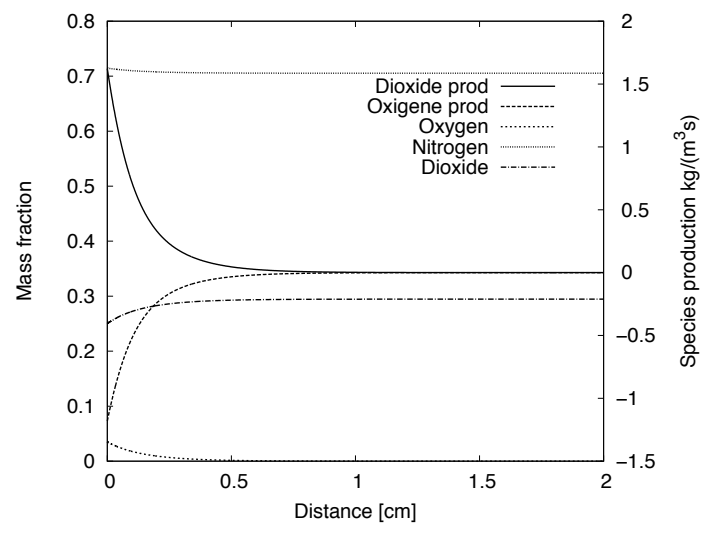

(a) 1 seconds

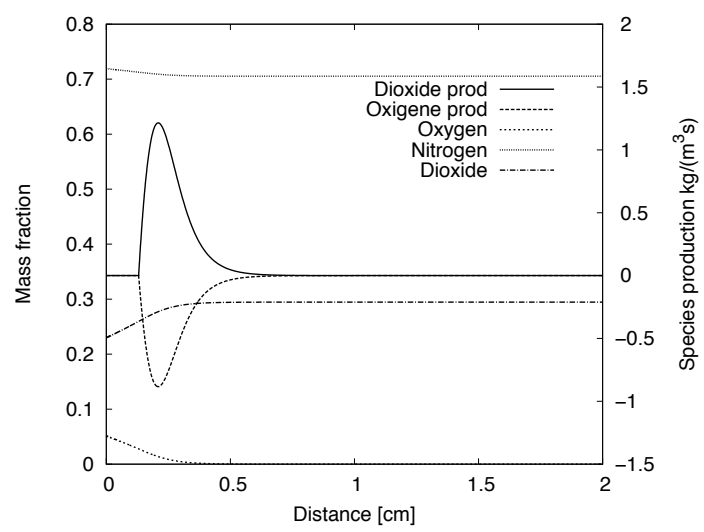

(c) 15 minutes

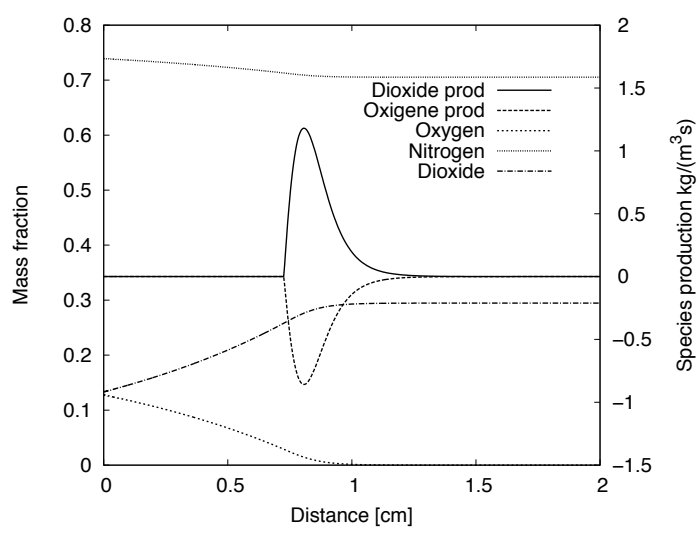

(e) 50 minutes

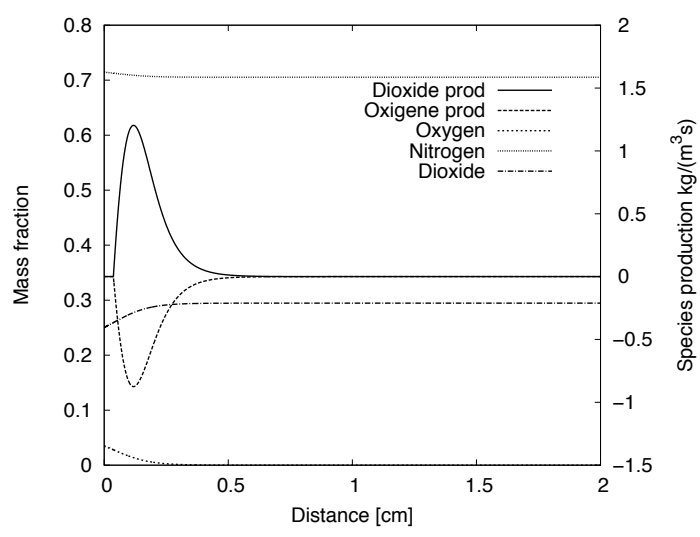

(b) 10 minutes

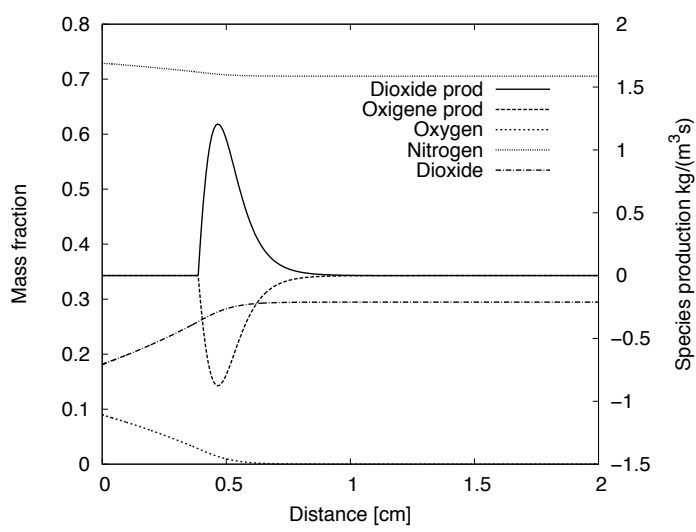

(d) 30 minutes

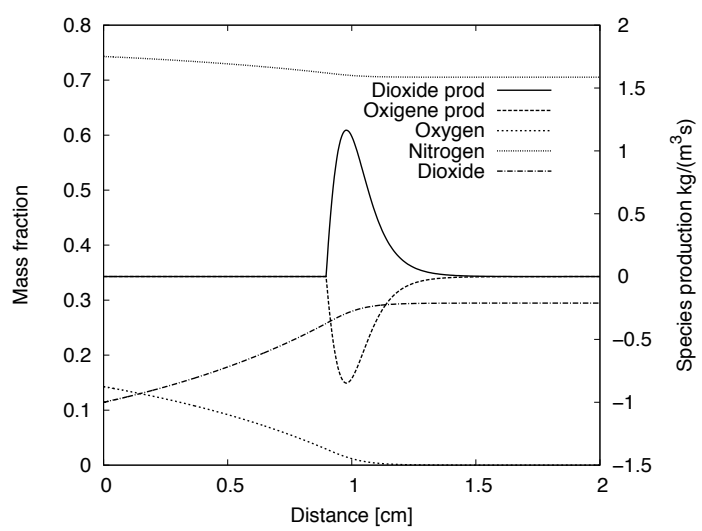

(f) 60 minutes

Figure 6. Time dependent gas mass fraction and species production rate inside the FiberForm sample for a mass flow rate of $2.149 \mathrm{mg} / \mathrm{s}$ and a variable fiber reactivity of $k_{f}=10^{5} e^{-1.2 \times 10^{5} /(R T)} \mathrm{m} / \mathrm{s}$ 


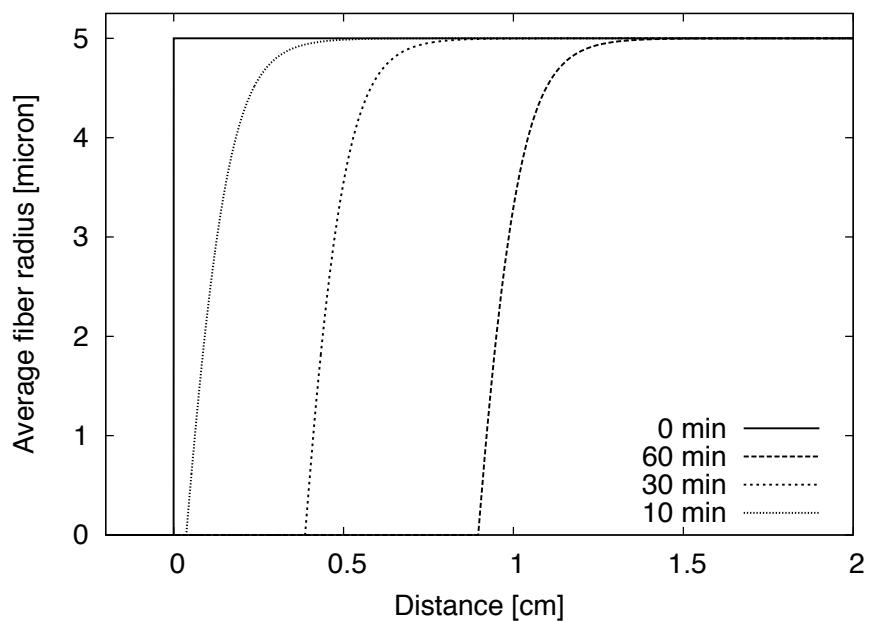

Figure 7. Time dependent fiber diameter inside the FiberForm sample for a mass flow rate of $2.149 \mathrm{mg} / \mathrm{s}$ and a variable fiber reactivity of $k_{f}=10^{5} e^{-1.2 \times 10^{5} /(R T)} \mathbf{m} / \mathbf{s}$

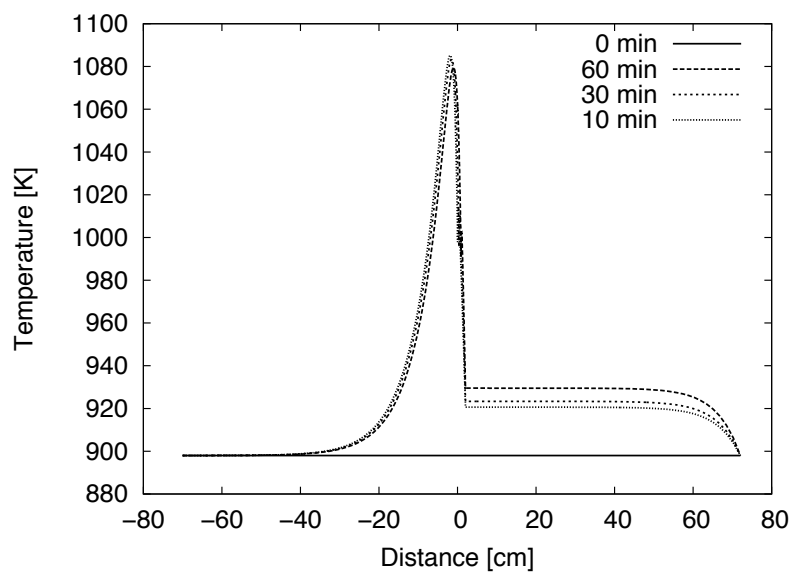

(a) Inside the flow tube reactor

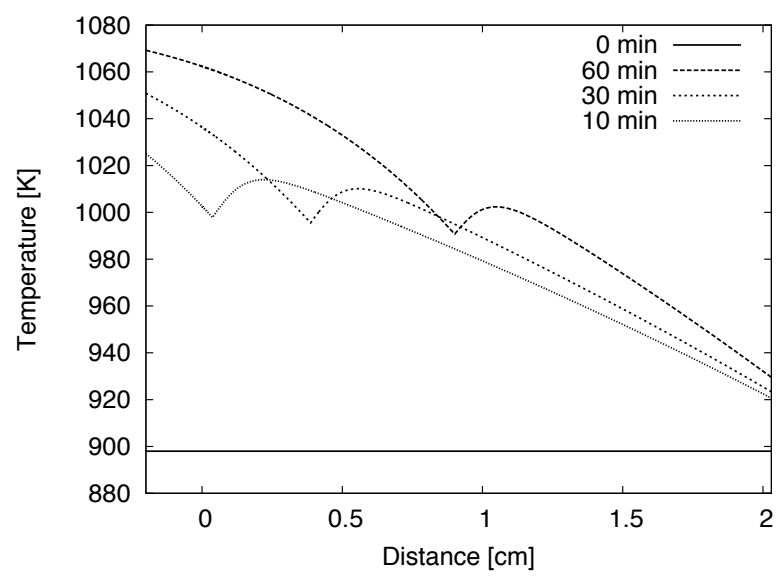

(b) Inside the FiberForm sample

Figure 8. Temperature as a function of time, with a variable fiber reactivity of $k_{f}=10^{5} e^{-1.2 \times 10^{5} /(R T)} \mathbf{m} / \mathbf{s}$ 